\title{
Development of nanostructure control process in the solution for application to energy and environmental fields
}

\author{
Eiji HOSONO
}

Energy Interface Technology Research Group, Energy Technology Research Institute, National Institute of Advanced Industrial Science and Technology, 1-1-1 Umezono, Tsukuba 305-8568, Japan

\begin{abstract}
The nanostructure control processes based on crystal growth in the solution are introduced. In the main, the self-template method is mentioned. The intermediate compounds such as metal hydroxides obtained by the crystal growth process play a role of selftemplate. After conversion of intermediate compounds with nanostructure such as nanosheet, the materials with the morphology, which reflects the morphology of the self-template, are obtained. The resultant materials are nanostructured materials with specific morphology. In addition, various nanostructured materials are fabricated by self-template methods through hydrothermal process or flux process. As the applications, dye-sensitized solar cells and Li ion batteries are measured. Moreover, the nanostructure control technique in the solution is used for not only electrochemical devices but also the fabrication of superhydrophobic films.
\end{abstract}

(C)2012 The Ceramic Society of Japan. All rights reserved.

Key-words : Nanostructure control, Crystal growth, Dye-sensitized solar cells, Superhydrophobic materials, Li ion battery

[Received October 11, 2011]

\section{Introduction}

In these days, the solvation of energy and environmental problems is big task for industrial and academic researchers. It is considered that nanostructure controlled materials can develop the performances of many devises. Therefore, the study of nanomaterials and control of nanostructure attracts many researchers in a lot of fields. Many review papers such as "Bioinspired ceramic thin film processing: Present status and future perspective", 1) "Powder-less processing for nano-structured bulk ceramics: Realization of direct fabrication from solutions and/or melts", "Liquid phase patterning of ceramics", 3) "Surfactant-free nonaqueous synthesis of metal oxide nanostructures", "Nanomaterials for rechargeable lithium batteries", "Controlling mineral morphologies and structures in biological and synthetic systems", ", "Synthesis of semiconducting functional materials in solution: From II-VI semiconductor to inorganic-organic hybrid semiconductor nanomaterials" "7) are reported. I have researched the fabrication process of nanomaterials by using solution method ${ }^{8)-40}$ such as chemical bath deposition (CBD) method ${ }^{8)-26)}$ and hydrothermal proc$\mathrm{ess}^{27)-34)}$ as one of researchers for nanomaterials. The CBD method is interesting method. The important point of CBD method is a control of supersaturation degree. If the supersaturation degree is high, the deposition of powders is caused by homogeneous nucleation. The control of the reaction such as hydrolysis by selection of $\mathrm{pH}$ and temperature results in low supersaturation condition. The low supersaturation condition causes the nucleation on the substrate by heterogeneous nucleation and growth of the crystals. The films on the substrate are directly obtained. Many kinds of materials are fabricated on the substrate without heating process using furnaces. Therefore, CBD method is kind process for the environments because the process, which is conducted at low temperature and pressure of near ambient, do not need large equipment with such as vacuum systems.

Corresponding author: E. Hosono; E-mail: e-hosono@aist.go.jp
When metal oxide materials are fabricated by CBD and hydrothermal process, the resultant morphology is based on the original crystal structure in many cases. The morphology of $\mathrm{ZnO}$ with hexagonal rod structure, ${ }^{41)}$ rutile $\mathrm{TiO}_{2}$ with rectangular parallelpiped rod structure, ${ }^{27)}$ and anatase $\mathrm{TiO}_{2}$ with octahedrallike structure ${ }^{30)}$ are reported. Although the using of additional agent into the solution can change the morphology, ${ }^{42)}$ I tried drastic morphology change. The process includes the crystal growth process of intermediate compounds. The materials are fabricated thorough the intermediate compounds. We called this method as self-template method.

I have studied the fabrication of nanomaterials by the solution process as core technique. The fabricated nanomaterials are used for applications such as dye-sensitized solar cells (DSC), ${ }^{10), 15), 19), 24)}$ superhydophobic films, ${ }^{16), 23), 25)}$ Li ion batteries, ${ }^{18), 20), 28), 31)-34), 38), 39)}$ as the energy and environmental materials. The high surface nanomaterials are useful for the electrochemical devices such as DSC because the electrode with high surface area can adsorb many dyes on the surface and increase the conversion efficiency by increasing of absorbed solar light. Of course, nanomaterials with short diffusion length of lithium ion are suitable for high power lithium ion battery. Moreover, rough structure constructed by nanomaterials is suitable for improvement of superhydrophobic property based on Casiee's equation. ${ }^{43)}$ In this paper, control method of nanomaterials and some device performances are introduced.

\section{Self-template methods}

The materials are fabricated thorough the intermediate compounds as self-templates. This is self-template method. For example, the nanostructured metal oxides are fabricated by pyrolysis reaction of metal hydroxides as intermediate compounds. ${ }^{9-15), 18), 19), 22), 24)}$ The metal hydroxide with layered crystal structure causes nanosheet morphology by CBD method. The metal hydroxides are easily converted into metal oxides by heating process. At that time, the nanosheet morphology is maintained. Moreover, each nanosheet is constructed by nano- 

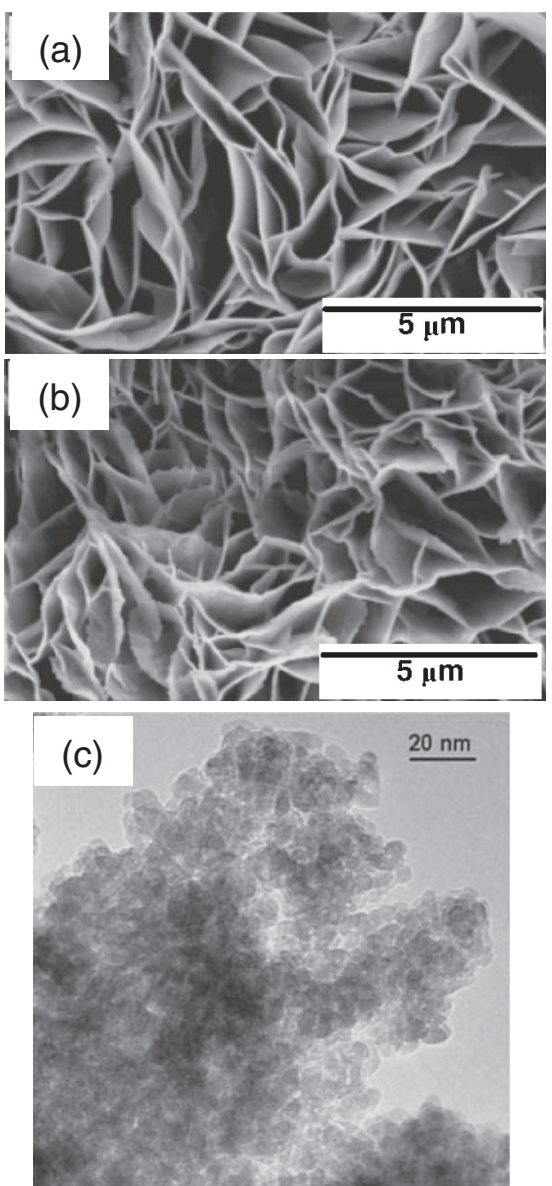

Fig. 1. (a) SEM image of the LHZA film. (b) SEM image of ZnO film obtained by heating of LHZA film. (c) TEM image of ZnO film obtained by heating of LHZA film. Copyright $($ C 2004, Elsevier.

particles. The resultant metal oxide films are high surface area materials.

At first, the fabrication of $\mathrm{ZnO}$ nanosheets is introduced. In the zinc acetate solution, the nanosheet film of $\mathrm{Zn}_{5}(\mathrm{OH})_{8}\left(\mathrm{CH}_{3} \mathrm{COO}\right)$. $2 \mathrm{H}_{2} \mathrm{O}$, which is one of layered hydroxide zinc acetate (LHZA), was obtained as shown in Fig. 1(a). ${ }^{11)}$ In order to obtain the morphology, the control of supersaturation degree for the heterogeneous nucleation and suitable construction of concentration gradient in the solution are important. After heating process, the LHZA was converted into $\mathrm{ZnO}$ with high surface area. The nanosheet morphology of LHZA was maintained as shown in Fig. 1(b). From Fig. 1(c), the nanoparticles constructed the nanosheet. This is a special feature of selftemplate method. The LHZA plays a role of self-template for the $\mathrm{ZnO}$ nanosheet morphology. Moreover, the self-template method through the chemical bath including zinc acetate and aluminum chloride resulted in Al-doped $\mathrm{ZnO}$ nanosheet film, which is relatively low resistivity, after heating. ${ }^{9)}$ It means that conductive nanosheet materials were fabricated by self-template method.

When cobalt acetate and nickel acetate were selected as the metal sources, layered hydroxide cobalt acetate and layered hydroxide nickel acetate films with nanosheet morphology in the solution were formed, respectively. After heating process, $\mathrm{Co}_{3} \mathrm{O}_{4}$ and $\mathrm{NiO}$ nanosheet films were obtained. ${ }^{13)}$

The self-template method is used not only metal oxide but also metal oxyfluoride materials. The addition of trifluoroacetic
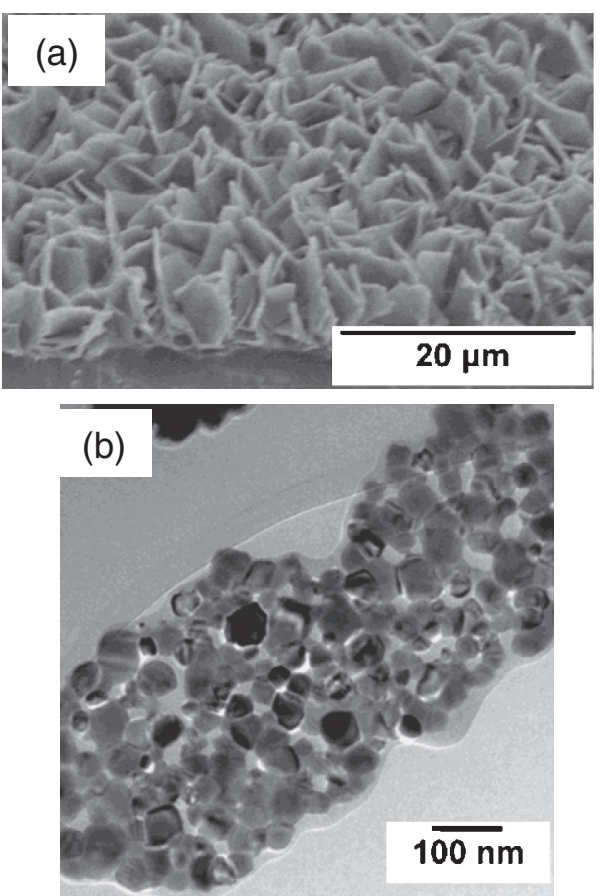

Fig. 2. SEM (a) and TEM (b) images of LaOF film fabricated by self-template method. Copyright 2004 Americal chemical society.

acid into the lanthanum acetate solution resulted in the lanthanum hydroxide films including acetate and trifluoroacetate groups. The sheet film was converted into the LaOF sheet ${ }^{12)}$ constructed by nanoparticles as shown in Figs. 2(a) and 2(b).

\section{DSC by self template method}

Generally, in chemical bath deposition, the film on surface facing the bottom of bottles grows only by heterogeneous nucleation. The film grown on the opposite side is composed of precipitates that are formed on the substrate as well as in the solution through the homogeneous nucleation as shown in Scheme 1. For getting thick film, the latter process is suitable. The $\mathrm{ZnO}$ nanosheet thick films were fabricated via LHZA thick films in Fig. 3(a). The thick films with high surface area were applied to the electrode of DSC. The DSC using the $\mathrm{ZnO}$ and Eosin $\mathrm{Y}$ as the dye indicated the conversion efficiency of $2 \%{ }^{10}$ )

The oriented $\mathrm{ZnO}$ nanosheet film ${ }^{15)}$ was fabricated thorough the oriented layered hydroxide zinc carbonate nanosheet film by self-template method as shown in Fig. 3(b). The DSC using the $\mathrm{ZnO}$ films with N-719 dye, which is one of the Ru dyes, showed the conversion efficiency of $3.9 \%$. The thickness of oriented nanosheet films was increased by repetition of crystal growth process. ${ }^{24)}$ The resultant thick ZnO oriented films with D-149 dye, which is organic dye without metal, indicated the conversion efficiency of over $4 \%$. These results indicate that the selftemplate method is useful for the electrochemical devices such as DSC because the electrode with high surface area can adsorb many dyes on the surface and increase the conversion efficiency by increasing of absorbed solar light.

\section{Other applications by the self template method}

In the $\mathrm{Li}$ ion battery as other electrochemical device, $\mathrm{NiO}$ nanomaterial by self-template method indicated good high power performance. ${ }^{18)}$ Moreover, $\mathrm{TiO}_{2}$ nanosheet films were fabricated ${ }^{29)}$ via heating process of ammonium titanate nanosheet film, which was fabricated by the hydrothermal process. It is one 


\section{Heterogeneous nucleation on the substrate and \\ Homogeneous nucleation in the solution}

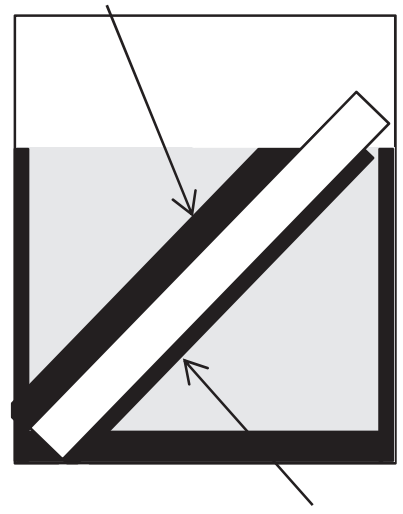

Heterogeneous nucleation

Scheme 1. Image of the chemical bath deposition method.
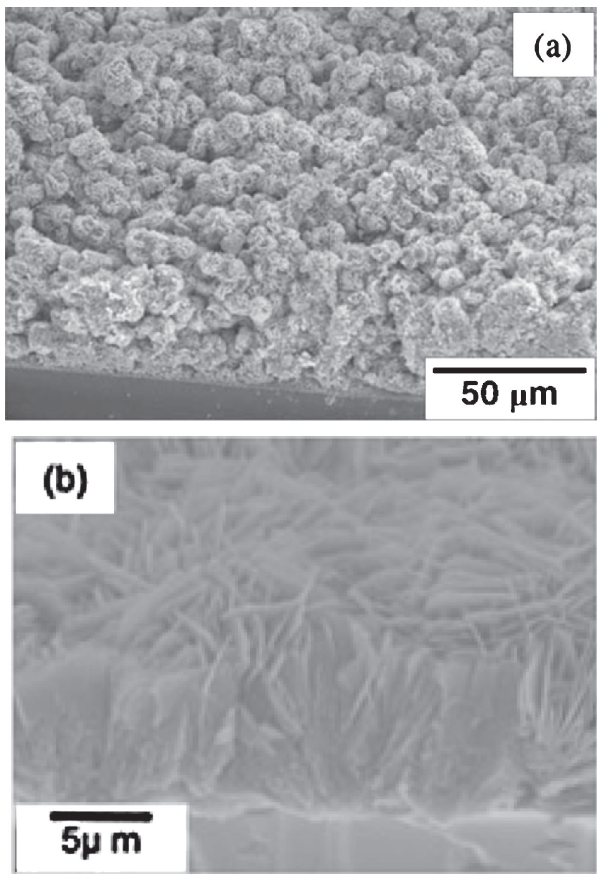

Fig. 3. (a) SEM image of the $\mathrm{ZnO}$ thick film. Copyright (C) 2004, Elsevier. (b) SEM image of oriented ZnO film. Copyright Ac 2005 WILEY-VCH Verlag GmbH \& Co. KGaA, Weinheim.

of the self-template methods. The $\mathrm{TiO}_{2}$ nanosheet showed the superhydrophilic property without UV irradiation. It is considered that self-template method is useful process for getting useful nanomaterials.

\section{Superhydrophobic property by using nanostructured film}

In this section, the superhydrophobic materials based on nanostructured film fabricated by crystal growth process in the solution are introduced. At first, the importance of nanostructured film is mentioned. In the Wenzel's equation, ${ }^{44)}$ when the surface is rough structure, the hydrophobicity of hydrophobic surface and hydrophilicity of hydrophilic surface are emphasized. It means nanostructure control is important for the hydrophobic and
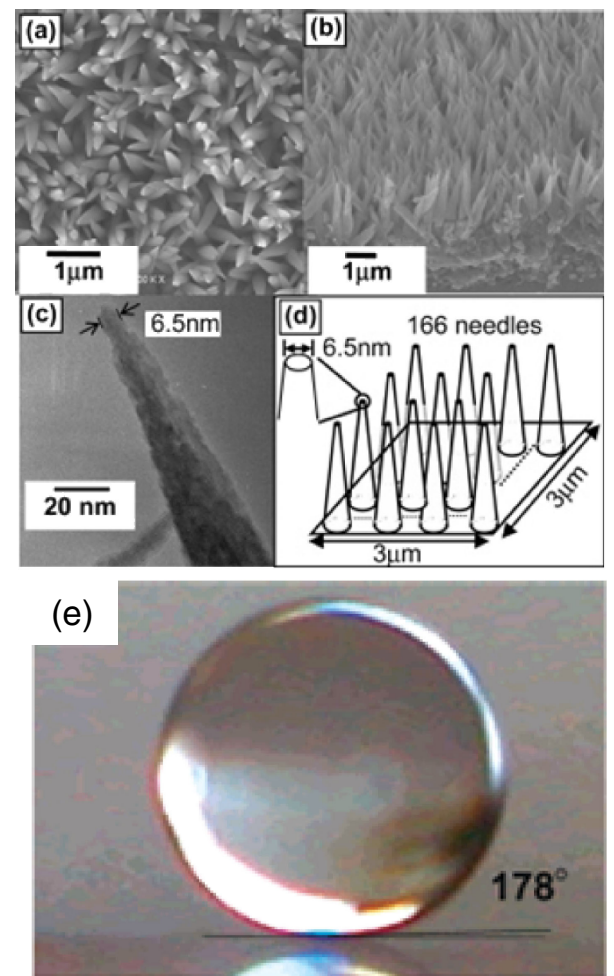

Fig. 4. (Color online) (a,b) SEM images of the nanopin films coated by lauric acid observed from the top and side, respectively. (c) TEM image of the nanopin film coated by lauric acid. (d) A simple model of the film. (e) The image of water on the superhydrophobic film. Copyright 2005 Americal chemical society.

hydrophilic property. On the other hands, Cassie's equation ${ }^{43)}$ takes account of trapped air in the rough structure. The equation is as follows.

$$
\cos \theta_{\mathrm{c}}=\Phi_{1} \cos \theta_{1}+\Phi_{2} \cos \theta_{2}
$$

$\theta_{\mathrm{c}}$ is a superficial contact angle; $\theta_{1}$ and $\theta_{2}$ are the contact angles of flat films of components 1 and 2, respectively. The $\Phi_{1}$ and $\Phi_{2}$ are surface area fractions of components 1 and 2, respectively.

When component 2 is the air component with the water contact angle of $180^{\circ}$, Eq. (1) is expressed as Eq. (2).

$$
\cos \theta_{\mathrm{c}}=\Phi_{1}\left(\cos \theta_{1}+1\right)-1
$$

This equation means that the surface with small $\Phi_{1}$ and large $\theta_{1}$ will improve superhydrophobic properties and the contact angle of water comes near to the air by suitable nanostructure control.

The cobalt hydroxide nanopin film, which was fabricated by CBD method, ${ }^{14}$ coated by the lauric acid was used for the measurement of superhydrophobic property. ${ }^{16)}$ The lauric acid is classified as hydrophilicity because the contact angle is less than 90 degree. From the SEM and TEM images in Figs. 4(a)-4(c), the nanostructure was modeled in Fig. 4(d). Therefore, the value of $\Phi_{1}$ was very small. By using Cassie's equation, the calculated contact angle was around $178^{\circ}$. When water was dropped on the surface of the film as shown in Fig. 4(e), the contact angle of $178^{\circ}$ was similar to the calculated value. So, the suitable nanostructure control results in the excellent superhydrophobic property.

The CBD method is suitable for the fabrication of superhydrophobic film because the film should be fabricated on the substrate with large area by low cost process for practical use. By using the oxygen-bubbled aqueous solution of manganese acetate as chemical bath, $\mathrm{MnOOH}$ nanorods films were easily fabri- 

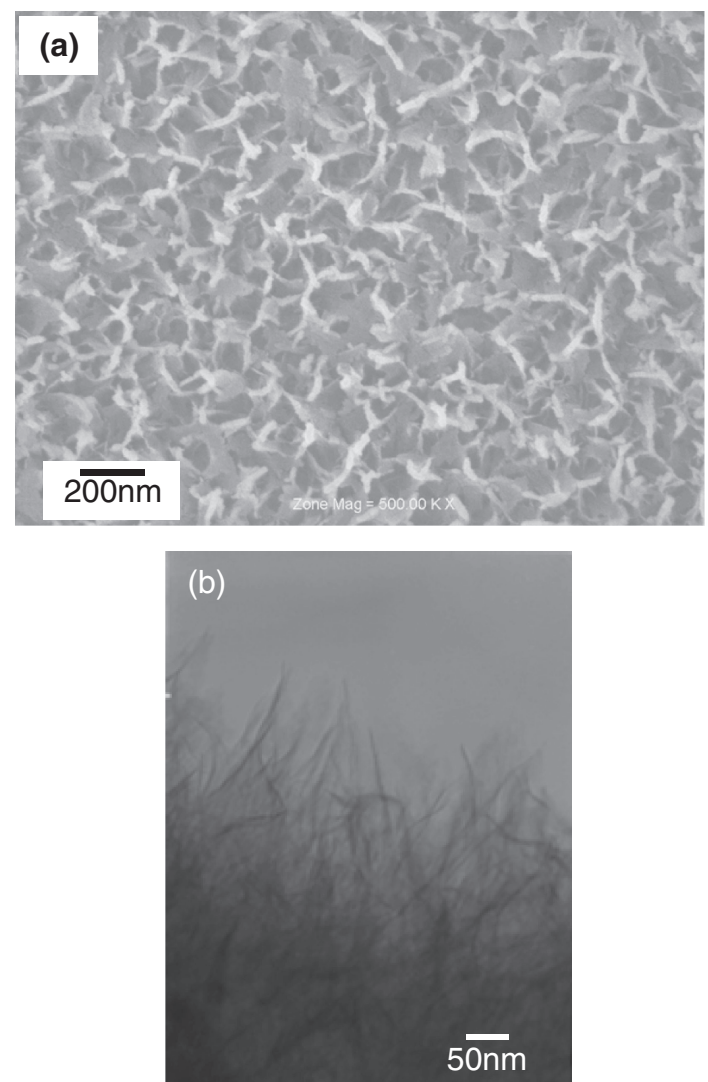

Fig. 5. SEM (a) and TEM (b) images of nanosheet films fabricated by hot water treatment of $\mathrm{Al} /$ glass substrate. Copyright 2009 Ceramic Society of Japan.

cated. ${ }^{23)}$ The coating of fluoroalklysilane indicated good superhydrophobic property. The hot water treatment of Al easily caused the nanosheet structured film. ${ }^{25)}$ Figs. 5(a) and 5(b) shows the SEM and TEM images of nanosheet film by the hot water treatment of $\mathrm{Al} /$ glass substrate, respectively. The coating of fluoroalklysilane resulted in good superhydrophobic property. The easy process for the nanostructured materials is suitable for the application to the superhydrophobic field.

\section{Li ion batteries by using nanowires materials}

For prevention of global warming, the development of the electric vehicle and plug-in hybrid vehicle to suppress discharge of $\mathrm{CO}_{2}$ is desired. As energy source of those next generation vehicles, $\mathrm{Li}$ ion batteries attract a great deal of public attention. In order to develop the battery performances, the study of large capacity and large power is important in the application to the vehicle. The large capacity materials have been researched in the world. Especially, there are clear problems to be solved to improve the power performances as follows. The particle size should be decreased to decrease the diffusion length of Li. The current density in the electrode should be decreased under the fast charge/discharge condition. Cycle performances should be improved under the fast charge/discharge condition. The electroconductivity of materials should be increased. In nanoparticles, the diffusion length of $\mathrm{Li}$ is shorter than that of bulk. The high surface area materials based on nanomaterials can decrease the current density. The nanomaterials caused relaxation of volume change due to the intercalation and deintercalation of $\mathrm{Li}$. It is related to the improvement of cycle performances. To improve the electroconductivity, some techniques such as mixing con-

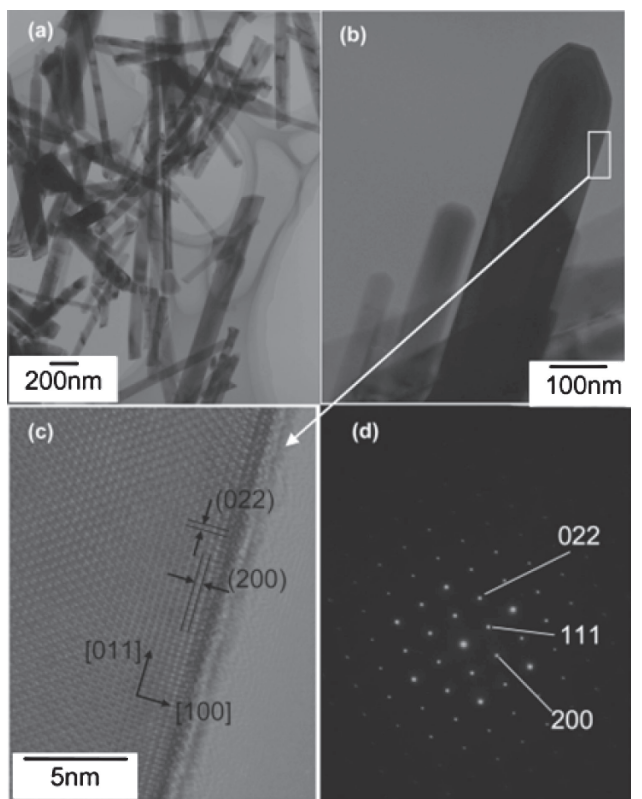

Fig. 6. (a) TEM images of the synthesized single crystalline spinel $\mathrm{LiMn}_{2} \mathrm{O}_{4}$ nanowires. (b, c) High-resolution TEM images of the nanowires. (d) Electron diffraction patterns of the nanowires. Copyright 2009 Americal chemical society.

ductive materials and nanomaterials, or coating of conductive materials on the nanomaterials are needed. Therefore, the using of nanomaterials to the high power batteries attracts many researchers and the control of nanostructure is important.

In these days, nanoparticulate materials are relatively easily fabricated by solution methods. However, nanoparticles with high surface activity are easy to aggregate. The many defects and low crystalinity are feared. Although high temperature heating is general process to improve the crystalinity of materials, the process easily causes the growth of nanoparticles and resultant particles are not nanoparticles. It is considered that the nanomaterials, which is not hard to aggregate, with high crystalinity is important for the development of high power Li ion battery. We expected that the nanomaterials with one dimensional wire structure are suitable morphology with high crystalinity.

We reported the fabrication of single crystalline $\mathrm{Na}_{0.44} \mathrm{MnO}_{2}$ nanowire ${ }^{31)}$ by hydrothermal process. It is considered that the one dimensional nanowire morphology is based on the crystal structure of tunnel structure. Single crystalline $\mathrm{Li}_{0.44} \mathrm{MnO}_{2}$ nanowire was fabricated by ion exchange reaction from $\mathrm{Na}_{0.44} \mathrm{MnO}_{2}$ nanowire as the template in the flux. ${ }^{34)}$ Moreover, single crystalline $\mathrm{LiMn}_{2} \mathrm{O}_{4}$, which is cubic spinel crystal structure, with nanowire morphology as shown in Fig. 6 was fabricated by heating process after flux reaction of $\mathrm{Na}_{0.44} \mathrm{MnO}_{2}$ nanowire as the template. ${ }^{32)}$ Generally, the fabrication of cubic crystal nanowire is difficult because the crystal structure is not anisotropy but isotropy. By using the self-template method, interesting materials were fabricated. The resultant $\mathrm{LiMn}_{2} \mathrm{O}_{4}$ nanowires showed good high rate performances.

\section{Conclusions}

The fabrication process of nanostructured materials were introduced in this paper. The processes using the crystal growth in the solution showed many kinds of nanostructured materials. The resultant nanomaterials were used for some applications such as, dye-sensitized solar cells, superhydrophobic property, and 
$\mathrm{Li}$ ion batteries. It is considered that these applications contribute to solve the earth-environmental problems. The nanostructure control is important for not only academic study but also industrial study.

Acknowledgment The author wishes to express his deepest gratitude to Professor Toshio Kimura, Associate Professor Shinobu Fujihara, Professor Hiroaki Imai of Keio University, Professor Itaru Honma of Tohoku University, Dr. Haoshen Zhou of National Institute of Advanced Industrial Science and Technology, Professor Tetsuichi Kudo of Tokyo University for their instructive discussions on this work.

\section{References}

1) Y. F. Gao and K. Koumoto, Cryst. Growth Des., 5, 1983-2017 (2005).

2) M. Yoshiumura, J. Ceram. Soc. Japan, 114, 888-895 (2006).

3) Y. Masuda, J. Ceram. Soc. Japan, 115, 101-109 (2007).

4) N. Pinna and M. Niederberger, Angew. Chem., Int. Ed., 47, 5292-5304 (2008).

5) P. G. Bruce, B. Scrosati and J.-M. Tarascon, Angew. Chem., Int. Ed., 47, 2930-2946 (2008).

6) F. C. Meldrum and H. Cölfen, Chem. Rev., 108, 4332-4432 (2008).

7) W. T. Yao and S. H. Yu, Adv. Funct. Mater., 18, 3357-3366 (2008).

8) E. Hosono, S. Fujihara and T. Kimura, Electrochem. SolidState Lett., 7, C49-C51 (2004).

9) E. Hosono, S. Fujihara and T. Kimura, J. Mater. Chem., 14, 881-886 (2004).

10) E. Hosono, S. Fujihara and T. Kimura, Electrochim. Acta, 49, 2287-2293 (2004).

11) E. Hosono, S. Fujihara, T. Kimura and H. Imai, J. Colloid Interface Sci., 272, 391-398 (2004).

12) E. Hosono, S. Fujihara and T. Kimura, Langmuir, 20, 37693774 (2004).

13) S. Fujihara, E. Hosono and T. Kimura, J. Sol-Gel Sci. Technol., 31, 165-168 (2004).

14) E. Hosono, S. Fujihara, I. Honma and H. S. Zhou, J. Mater. Chem., 15, 1938-1945 (2005).

15) E. Hosono, S. Fujihara, I. Honma and H. S. Zhou, Adv. Mater., 17, 2091-2094 (2005).

16) E. Hosono, S. Fujihara, I. Honma and H. S. Zhou, J. Am. Chem. Soc., 127, 13458-13459 (2005).

17) S. Fujihara, Y. Shibata and E. Hosono, J. Electrochem. Soc., 152, C764-C768 (2005).

18) E. Hosono, S. Fujihara, I. Honma and H. S. Zhou, Electrochem. Commun., 8, 284-288 (2006).

19) K. Kakiuchi, E. Hosono and S. Fujihara, J. Photochem.
Photobiol., A, 179, 81-86 (2006).

20) E. Hosono, S. Fujihara, I. Honma, M. Ichihara and H. S. Zhou, J. Electrochem. Soc., 153, A1273-A1278 (2006).

21) E. Hosono, S. Fujihara, I. Honma, M. Ichihara and H. S. Zhou, J. Power Sources, 158, 779-783 (2006).

22) K. Kakiuchi, E. Hosono, T. Kimura, H. Imai and S. Fujihara, J. Sol-Gel Sci. Technol., 39, 63-72 (2006).

23) E. Hosono, M. Ichihara and H. S. Zhou, Nanotechnology, 19, 395605 (2008).

24) E. Hosono, Y. Mitsui and H. S. Zhou, Dalton Trans., 40, 54395441 (2008).

25) E. Hosono, Y. R. Wang, M. Ichihara and H. S. Zhou, J. Ceram. Soc. Japan, 117, 299-301 (2009).

26) K. Sakaushi, Y. Oaki, H. Uchiyama, E. Hosono, H. S. Zhou and H. Imai, Small, 6, 776-781 (2010).

27) E. Hosono, S. Fujihara, K. Kakiuchi and H. Imai, J. Am. Chem. Soc., 126, 7790-7791 (2004).

28) M. Okubo, E. Hosono, J. Kim, M. Enomoto, N. Kojima, T. Kudo, H. S. Zhou and I. Honma, J. Am. Chem. Soc., 129, 7444-7452 (2007).

29) E. Hosono, H. Matsuda, I. Honma, M. Ichihara and H. S. Zhou, Langmuir, 23, 7447-7450 (2007).

30) E. Hosono, S. Fujihara, H. Imai, I. Honma, I. Masaki and H. S. Zhou, ACS Nano, 1, 273-278 (2007).

31) E. Hosono, H. Matsuda, I. Honma, S. Fujihara, M. Ichihara and H. S. Zhou, J. Power Sources, 182, 349-352 (2008).

32) E. Hosono, T. Kudo, I. Honma, H. Matsuda and H. S. Zhou, Nano Lett., 9, 1045-1051 (2009).

33) M. Okubo, Y. Mizuno, H. Yamada, J. Kim, E. Hosono, H. S. Zhou, T. Kudo and I. Honma, ACS Nano, 4, 741-752 (2010).

34) E. Hosono, H. Matsuda, T. Saito, T. Kudo, M. Ichihara, I. Honma and H. S. Zhou, J. Power Sources, 195, 7098-7101 (2010).

35) E. Hosono, S. Fujihara, T. Kimura and H. Imai, J. Sol-Gel Sci. Technol., 29, 71-79 (2004).

36) E. Hosono and S. Fujihara, Chem. Comm., 2062-2063 (2004).

37) E. Hosono, S. Fujihara, H. Imai, I. Honma and H. S. Zhou, Chem. Commun., 20, 2609-2611 (2005).

38) E. Hosono, H. Matsuda, I. Honma, M. Ichihara and H. S. Zhou, J. Electrochem. Soc., 154, A146-A149 (2007).

39) Y. G. Wang, Y. R. Wang, E. Hosono, K. X. Wang and H. S. Zhou, Angew. Chem., Int. Ed., 47, 7461-7465 (2008).

40) K. Sakaushi, Y. Oaki, H. Uchiyama, E. Hosono, H. S. Zhou and H. Imai, Nanoscale, 2, 2424-2430 (2010).

41) S. Yamabi and H. Imai, J. Mater. Chem., 12, 3773-3778 (2002).

42) J. Yahiro, Y. Oaki and H. Imai, small, 2, 1183-1187 (2006).

43) A. B. D. Cassie, Discuss. Faraday Soc., 3, 11-16 (1948).

44) R. N. Wentzel, J. Phys. Colloid Chem., 53, 1466-1467 (1949).

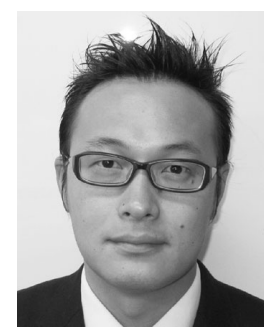

Eiji Hosono received the Ph.D. at Keio University in 2004. From 2004 to 2007, he joined Energy Technology Research Institute, National Institute of Advanced Industrial Science and Technology (AIST) as postdoctoral fellow of Japan Science and Technology Agency (JST) or Japan Society for the Promotion of Science (JSPS). After that he was employed as a researcher in Energy Technology Research Institute, AIST. His current research is the fabrication of advanced functional materials such as lithium ion batteries and superhydrophobic films based on the nanostructure control. 